\title{
Factors Associated with Survival in Patients Undergoing Invasive Mechanical Ventilation in an Intensive Care Unit in Colombia, 2017-2018: A Retrospective Cohort Study
}

\author{
Jorge Enrique Machado-Alba ${ }^{1}$ [ - Andrés Felipe Usma-Valencia ${ }^{1} \cdot$ Nicolás Sánchez-Ramírez $^{1}$. \\ Luis Fernando Valladales-Restrepo ${ }^{1,2}(\mathbb{D})$ Manuel Machado-Duque ${ }^{1,2}\left(\mathbb{D} \cdot\right.$ Andrés Gaviria-Mendoza $^{1,2}(\mathbb{D}$
}

Accepted: 28 February 2021 / Published online: 7 April 2021

(c) The Author(s) 2021

\begin{abstract}
Background Critically ill patients are admitted to intensive care units so they can be comprehensively managed and provided with services not covered in general hospital wards, with the aim to increase their chances of survival. These procedures include invasive mechanical ventilation.

Objective The aim of this study was to identify the factors associated with survival in critically ill patients who required invasive mechanical ventilation in an intensive care unit of a tertiary-level hospital in Colombia.

Methods This was a retrospective follow-up study of a cohort of adult patients who required invasive mechanical ventilation in an intensive care unit in San José de Buga Hospital, between 2017 and 2018. Sociodemographic, clinical, and pharmacological variables were identified. Using Cox regression, variables associated with survival and complications were identified. Results A total of 357 patients were analyzed. The average age was $64.8 \pm 18.9$ years, and $52.9 \%$ were male. The most frequent diagnoses were sepsis/septic shock (38.4\%) and trauma (17.4\%). The main factors associated with shorter survival were advanced age (HR 0.97; 95\% CI 0.96-0.99), a diagnosis of septic shock (HR 0.29; 95\% CI 0.18-0.48) or diabetes mellitus at admission (HR 0.57; 95\% CI 0.33-0.98), a healthcare-associated infection (HR 0.51; 95\% CI 0.33-0.80), and the need for vasopressors (HR 0.36; 95\% CI 0.22-0.59). The administration of systemic corticosteroids was associated with a higher probability of survival (HR 1.93; 95\% CI 1.15-3.25).

Conclusions The use of systemic corticosteroids was associated with a greater probability of survival in critically ill patients who required invasive mechanical ventilation in an intensive care unit. The identification of the variables associated with a higher risk of dying should allow care protocols to be improved, thereby extending the life expectancy of these patients.
\end{abstract}

Jorge Enrique Machado-Alba

machado@utp.edu.co

1 Grupo de Investigación en Farmacoepidemiología y Farmacovigilancia, Universidad Tecnológica de PereiraAudifarma S.A, Calle 105 No. 14-140, 660003 Pereira, Risaralda, Colombia

2 Grupo de Investigación Biomedicina, Facultad de Medicina, Fundación Universitaria Autónoma de las Américas, Pereira, Colombia

\section{Key Points}

Advanced age was associated with shorter survival in patients admitted to the intensive care unit.

Patients who were admitted to the ICU with a diagnosis of septic shock, who had a history of diabetes mellitus, and those who developed a hospital infection were less likely to survive.

The administration of systemic corticosteroids was associated with an increased probability of survival. 


\section{Introduction}

Critically ill patients are admitted to intensive care units (ICUs) to comprehensively manage them and provide them with services not covered in general hospital wards, aiming to increase their chances of survival [1]. One of the main services provided by ICUs, unlike other wards, is mechanical ventilation, both invasive and noninvasive, which can have many indications in both surgical and medical contexts. In the latter are those causes that generate respiratory failure, where etiologies such as pneumonia and chronic obstructive pulmonary disease (COPD) predominate, and causes of cardiovascular and neurological origin, where heart failure predominates [2, 3]. However, this type of intervention can be quite challenging despite its obvious benefits, both due to the need to sedate the patient during treatment and due to the complications inherent to the increased risk of healthcare-associated infections (HAIs) and the difficulties that may arise from prolonged mechanical ventilation and extubation failure $[4,5]$.

Before the start of the coronavirus disease pandemic (COVID-19), it was estimated that 5 million people were admitted to the ICU in the United States each year, with $20-40 \%$ of patients requiring mechanical ventilation and their annual mortality being between 10 and $29 \%$ according to age. Sepsis was the most common cause of admission and carried an annual cost of approximately 2 billion dollars [6]. However, recently the respiratory illness caused by severe acute respiratory syndrome coronavirus 2 (SARS-CoV-2) has led to a large occupancy of ICUs, which was unprecedented worldwide, with large numbers of patients presenting severe respiratory failure, many of whom have required interventions by mechanical ventilation [7], and presenting a mortality of $35.5-41.6 \%$ [8]. ICU admission strategies can put a high burden on society and the health system, generating ethical/moral conflicts about the cost effectiveness of certain therapies and lengthy stays involving these services [9].

Survival or mortality depends not only on the pathology at admission but also on such factors as the magnitude of the disease, the occurrence of a rapid and efficient response at the time of the intervention, and sociodemographic characteristics (i.e., age and sex) etc. $[1,10]$. Among the variables related to mortality are the presence of comorbidities, a high Charlson index $(>2)$, sepsis/ septic shock, and advanced age $[11,12]$. In contrast, factors associated with survival are a tertiary level of care, rapid response, young age, being female, and having had fewer comorbidities or a low Charlson index [ $\leq 2]$ [11]. The knowledge of these survival and mortality factors can impact the decision made based on the prognosis of each patient under the ICU's admission strategy and the decision made about mechanical ventilation, and such knowledge can also help control costs for the health system and advise the implementation of therapeutic strategies that may benefit patients [13].

Therefore, the aim of this study was to identify the factors associated with survival in critical patients who required invasive mechanical ventilation in an intensive care unit of a high-complexity hospital during the years 2017 and 2018 in Colombia.

\section{Materials and Methods}

A retrospective observational study of patients requiring invasive mechanical ventilation in an ICU was performed by analyzing their sociodemographic, clinical, comedication, and other pharmacological variables, as well as complications and factors associated with their survival, from electronic medical records and a drug dispensing database. We followed up a cohort of patients who were treated between January 1, 2017 and December 31, 2018 in the ICU of the Fundación Hospital San José de Buga (San José de Buga Hospital) in the Valle del Cauca, Colombia. This is a tertiary-level hospital that treats patients affiliated with the contributory (employer-paid) regime as well as the state-subsidized regime within Colombia's Health System. The follow-up of each patient began from the time they were admitted to the hospital and, especially, from their admission to the ICU until they were discharged from the clinic, were referred to another hospital, or died.

Patients of either sex aged 18 years or older, who required invasive mechanical ventilation therapy in the ICU and who had complete medical records were enrolled. All the medical records of the established period were reviewed considering the inclusion (those over 18 years of age, of any sex, on invasive mechanical ventilation, receiving sedation with midazolam or dexmedetomidine or both and who had complete clinical records) and exclusion criteria (incomplete clinical records). The research was conducted in a time period preceding the COVID-19 pandemic.

From the information provided by the San José de Buga Hospital and the clinical and dispensing records during hospitalization, the data of interest were extracted, and a database was designed that allowed us to collect the following groups of variables:

1. Sociodemographic: sex, age, origin, occupation, and insurance affiliation regime.

2. Clinical: 
a. Physiological variables: heart rate, respiratory rate, blood pressure, temperature before invasive mechanical ventilation and after initiating sedation.

b. Comorbidities: admission diagnosis, diagnosis associated with mechanical ventilation, presence of other comorbidities. The Charlson comorbidity index was calculated.

c. Ventilation complications: infectious, tracheostomy, delirium, other.

d. Hospital stay: length of stay in the San José de Buga hospital (days), length of stay in the ICU, duration of mechanical ventilation, duration of weaning from mechanical ventilation.

e. History of mechanical ventilation.

f. Vital status: vital status at patient discharge (alive, dead)

3. Pharmacological:

a. Sedatives used: midazolam, dexmedetomidine, other (propofol, fentanyl). The use of midazolam as monotherapy and midazolam + dexmedetomidine combined was identified.

b. Comedications: vasopressors/inotropes (norepinephrine, adrenaline, dopamine, dobutamine), vasodilators (nitroprusside, nitroglycerin, other), opioids (morphine, fentanyl, other), antiepileptics (phenobarbital, phenytoin, valproic acid, levetiracetam, other), benzodiazepines (lorazepam, diazepam, clonazepam, other), antipsychotics (haloperidol, levomepromazine, olanzapine, quetiapine, others), antibacterials (penicillins, cephalosporins, monobactams, carbapenems, aminoglycosides, macrolides, tetracyclines, fluoroquinolones, other), insulins (human, analog), systemic corticosteroids (methylprednisolone, dexamethasone, betamethasone, hydrocortisone, other), antiulcer drugs (proton pump inhibitors, antihistaminergic $\mathrm{H} 2$, other), anticoagulants (unfractionated heparin, low-molecularweight heparins, other), antiplatelets (ASA, P2Y12 receptor antagonists, other), fibrinolytics (streptokinase, alteplase, other), others.

The protocol was approved by the Bioethics Committee of the Universidad Tecnológica de Pereira in the category of risk-free research. The ethical principles established by the Declaration of Helsinki were respected. Reference number: 01-220719.

The main outcome was the status at discharge (alive or dead). Secondary outcomes were the need for sedation with midazolam or dexmedetomidine during invasive mechanical ventilation and the presentation of complications.
The database was first searched for inconsistent data and extraneous or extreme values. A second search of the records was performed for missing data to corroborate them. The information was analyzed with the statistical package SPSS Statistics, version 26.0 for Windows (IBM, USA). A descriptive analysis was performed with frequencies and proportions for the qualitative variables and measures of central tendency and dispersion for the quantitative variables. Quantitative variables were compared through Student's $t$ test or ANOVA and through the $\chi^{2}$ test for categorical variables to find any associations with the primary outcome (being alive at hospital discharge). A Cox regression analysis was performed, selecting as outcome being alive at hospital discharge (survival), taking the duration of hospitalization in days as the follow-up time. The covariates were sex, age, and other associated variables in the bivariate models. Patients with referrals to another hospital center were censored, and follow-up was stopped on the day of the patient's discharge or death. A level of statistical significance of $p<0.05$ was used.

\section{Results}

A total of 357 patients were analyzed, with an average age of 64.8 years, mostly male and almost all from the department of Valle del Cauca (Colombia) $(n=353 ; 98.9 \%)$. Table 1 shows the sociodemographic characteristics and comorbidities of the patients.

Sixty-seven (18.8\%) patients had a single main diagnosis, $172(48.2 \%)$ had two diagnoses, and 118 (33.1\%) three diagnoses, the most frequent being sepsis/septic shock, followed by trauma and heart failure. Table 1 shows the main diagnoses at admission and Table 2 presents the medications used by these patients.

\subsection{Outcomes}

Of individuals who underwent mechanical ventilation, $65.8 \%(n=235)$ died during hospitalization, after a mean of $13.1 \pm 13.9$ days of total hospitalization and $9.1 \pm 11.1$ days in the ICU. All patients received sedation with midazolam, and in a third this was in combination with dexmedetomidine. At least one complication was identified in 104 (29.1\%) of the patients. Table 3 shows the results of the variables of hospitalization, sedation, complications, and clinical parameters of the patients evaluated.

\subsection{Multivariate Analysis}

The Cox proportional hazard regression model that was adjusted for age, admission diagnoses, comorbidities, use of sedation, and comedications, evaluating those variables 
Table 1 Sociodemographic characteristics and comorbidities in patients undergoing invasive mechanical ventilation admitted to an intensive care unit in Colombia, 2017-2018

\begin{tabular}{|c|c|c|}
\hline Variables & $N=357$ & $\%$ \\
\hline \multicolumn{3}{|l|}{ Demographics } \\
\hline Sex, male & 189 & 52.9 \\
\hline Age in years, mean (SD) & $64.8(18.9)$ & \\
\hline 18-39 years & 47 & 13.2 \\
\hline 40-64 years & 106 & 29.7 \\
\hline $65-79$ years & 123 & 34.5 \\
\hline$\geq 80$ years & 81 & 22.7 \\
\hline \multicolumn{3}{|l|}{ Insurance scheme } \\
\hline Contributory & 175 & 49.0 \\
\hline Subsidized & 155 & 43.4 \\
\hline Other & 27 & 7.6 \\
\hline \multicolumn{3}{|l|}{ Comorbidities (admission) } \\
\hline Charlson index, mean (SD) & $3.6(2.6)$ & \\
\hline Charlson index $\leq 2$ points & 127 & 35.6 \\
\hline Charlson index $>2$ points & 230 & 64.4 \\
\hline Hypertension & 212 & 59.4 \\
\hline COPD & 118 & 33.1 \\
\hline Diabetes mellitus & 100 & 28 \\
\hline Chronic kidney disease & 60 & 16.8 \\
\hline Ischemic heart disease & 60 & 16.8 \\
\hline Congestive heart failure & 42 & 11.8 \\
\hline Cerebrovascular disease & 27 & 7.6 \\
\hline Cancer & 16 & 4.5 \\
\hline Dementia & 9 & 2.5 \\
\hline Obesity & 9 & 2.5 \\
\hline Epilepsy & 7 & 2.0 \\
\hline Cirrhosis & 6 & 1.7 \\
\hline Dyslipidemia & 6 & 1.7 \\
\hline Benign prostatic hypertrophy & 6 & 1.7 \\
\hline Asthma & 5 & 1.4 \\
\hline Peripheral arterial disease & 4 & 1.1 \\
\hline Parkinson's disease & 2 & 0.6 \\
\hline \multicolumn{3}{|l|}{ Clinical features } \\
\hline \multicolumn{3}{|l|}{ Number of diagnoses (admission) } \\
\hline 1 & 67 & 18.8 \\
\hline 2 & 172 & 48.2 \\
\hline 3 & 118 & 33.1 \\
\hline \multicolumn{3}{|l|}{ Admission diagnosis } \\
\hline Septic shock & 137 & 38.4 \\
\hline Trauma & 62 & 17.4 \\
\hline Decompensated heart failure & 57 & 16.0 \\
\hline Community acquired pneumonia & 54 & 15.1 \\
\hline Asthma/COPD decompensation & 54 & 15.1 \\
\hline Abdominal infection/pathology & 52 & 14.6 \\
\hline Other shocks (different origin) & 52 & 14.6 \\
\hline Acute myocardial infarction & 39 & 10.9 \\
\hline Central nervous system bleeding & 29 & 8.1 \\
\hline Cardiogenic shock & 24 & 6.7 \\
\hline
\end{tabular}

Table 1 (continued)

\begin{tabular}{|c|c|c|}
\hline Variables & $N=357$ & $\%$ \\
\hline Asystolia/pulseless electrical activity & 18 & 5.0 \\
\hline Neurological pathology $\mathrm{y}^{\mathrm{a}}$ & 16 & 4.5 \\
\hline Other respiratory diseases & 16 & 4.5 \\
\hline Firearm or stab wounds & 13 & 3.6 \\
\hline Cancer-related complications & 13 & 3.6 \\
\hline Acute complications of diabetes & 12 & 3.4 \\
\hline Poisonings & 9 & 2.5 \\
\hline Supraventricular tachyarrhythmias ${ }^{\mathrm{b}}$ & 9 & 2.5 \\
\hline Ventricular tachyarrhythmias & 5 & 1.4 \\
\hline Bradyarrhythmias & 5 & 1.4 \\
\hline \multicolumn{3}{|c|}{$\begin{array}{l}C O P D \text { chronic obstructive pulmonary disease, } S D \text { standard deviation } \\
\text { a Stroke, encephalopathy, convulsive syndrome, neuropathies }\end{array}$} \\
\hline
\end{tabular}

that could be related during hospitalization with the outcome of being alive at discharge, found that the use of systemic glucocorticoids was associated with a greater probability of being discharged alive, while advanced age, an admission diagnosis of septic shock, suffering complications that required a tracheostomy, suffering an HAI, having diabetes mellitus, and requiring support with vasopressors/inotropes was associated with a lower probability of survival. No association was found with survival according to the type of sedation used, either dexmedetomidine + midazolam or midazolam alone (see Table 4).

\section{Discussion}

The present study identified factors related with a higher or lower probability of survival in a group of patients older than 18 years affiliated with the Colombian Health System who required invasive mechanical ventilation in an ICU. Invasive mechanical ventilation was more common in men and in those who had a diagnosis related to sepsis/septic shock or trauma, all in line with the regional literature, which indicates sepsis as one of the main causes of admission to the ICU $[1,14]$. Other studies that have evaluated the characteristics of patients admitted for sepsis/septic shock or trauma have found a predominance of males $[15,16]$.

Mortality in the ICU in this region is 30-50\% [1, 10], and in Europe it is 6.7-17.8\%, with exceptional cases such as that found in Poland where mortality was $42 \%$ [11], which is slightly higher than that found in this report. These patients had certain pathological antecedents that could increase the risk of mortality, such as diabetes mellitus, but the presence of this comorbidity has not been associated with an increased risk in other studies $[17,18]$. On the other hand, 
Table 2 Medications used by patients undergoing invasive mechanical ventilation admitted to an intensive care unit in Colombia, 20172018

\begin{tabular}{lll}
\hline Drugs & $n$ & $\%$ \\
& $(N=357)$ & \\
\hline
\end{tabular}

Analgesics and adjuvants

Neuromuscular relaxants

Non-opioid pain relievers

Opioid pain relievers

Ketamine

Thiopental

Cardiovascular

Parenteral anticoagulants

Vasopressors/inotropics

Diuretics

Antihypertensive

Atropine

Vasodilators

Antiarrhythmics

Nitrates

Oral anticoagulants

Fibrinolytics

Psychopharmaceuticals

Antiepileptic drugs

Antipsychotics

Benzodiazepines

Antidepressants

Neostigmine

Biperiden

Antidementia drugs

Endocrine system

Systemic corticosteroid

Lipid-lowering

Insulins

Levothyroxine

Oral antidiabetic

Antithyroid

Vitamin K

Antimicrobial or antiviral antibacterials

Use of 0-2 antibacterials or antivirals

Use of $>2$ antibacterials or antivirals

Antimicrobials or antivirals used

Cephalosporins

Carbapenems

Vancomycin

Aminoglycosides

Aminopenicillins

Macrolides

Piperacillin-tazobactam

Antifungals

Clindamycin

Linezolid
274

223

118

23

3

76.8

62.5

33.1

6.4

0.8

88.2

88.2

51.0

47.6

16.5

14.6

13.4

2.8

1.7

0.6

22.7

18.8

16.2

4.8

1.7

1.1

0.3

68.1

37.3

13.2

10.4

2.5

0.6

3.4

81.8

47.3

52.7

188

184

182

174

86

86

67

65

59

28

24
Table 2 (continued)

\begin{tabular}{lll}
\hline Drugs & $n$ & $\%$ \\
& $(N=357)$ & \\
\hline Nitroimidazoles & 16 & 4.5 \\
Quinolones & 13 & 3.6 \\
Trimethoprim/sulfamethoxazole & 12 & 3.4 \\
Colistin & 8 & 2.2 \\
Aztreonam (monobactam) & 7 & 2.0 \\
Antiviral & 5 & 1.4 \\
Tetracyclines & 3 & 0.8 \\
Tigecycline & 1 & 0.3 \\
Other medications & & \\
Proton-pump inhibitor & 347 & 97.2 \\
Laxatives & 192 & 53.8 \\
Inhaled bronchodilators and corticosteroids & 172 & 48.2 \\
Antiemetics & 118 & 33.1 \\
Antispasmodics & 62 & 17.4 \\
Antidiarrheal & 13 & 3.6 \\
Antihistamines & 9 & 2.5 \\
Albumin & 8 & 2.2 \\
\hline
\end{tabular}

in this study, a high Charlson index was not associated with a lower probability of survival, despite reports that have found such an association [12]. Similarly, admission to the ICU itself can already predispose to a lower patient survival. The ICE-CUB 2 study, which analyzed whether systematic admission (with greater flexibility) to the ICU in the elderly population had an impact on mortality, showed that patients older than 75 years who were systematically admitted to the ICU had a higher in-hospital mortality than patients of the same profile admitted to hospitals that did not have systematic ICU admission [19]. Likewise, advanced age has been a risk factor for increased mortality in the ICU [20], in line with this analysis, where a lower probability of survival with older age was observed.

Septic shock is a risk factor for in-hospital death. Worldwide, the mortality of hospitalized patients with sepsis/septic shock is $30-40 \%$ [21, 22], and in Colombia, it is approximately $33 \%$ [15], which is high despite therapeutic efforts, and more so in patients who receive invasive mechanical ventilation, which is a population at high risk of complications such as cardiovascular collapse and cardiac arrest [23]. In the post-hoc analysis of the SEPSISPAM study, it was found that mortality, as well as vasopressor therapy and hospitalization time, was significantly higher in patients with sepsis who were intubated than in those who were not [24].

Higher mortality has also been observed in those patients with HAIs, the most common (20-25\%) being ventilatorassociated pneumonia (VAP) $[5,25,26]$, which has been associated with higher lethality (OR 2.5; 95\% CI 1.5-4.1) [5] in line with the data found in this study. 
Table 3 Outcomes, type of sedation, and several clinical variables in patients undergoing invasive mechanical ventilation admitted to an intensive care unit in Colombia, 2017-2018

\begin{tabular}{|c|c|c|}
\hline Characteristics & $\begin{array}{l}\text { Frequency } \\
(N=357)\end{array}$ & $\%$ \\
\hline \multicolumn{3}{|l|}{ Outcome at discharge } \\
\hline Alive & 122 & 34.2 \\
\hline Dead & 235 & 65.8 \\
\hline \multicolumn{3}{|l|}{ Medications for sedation } \\
\hline Midazolam alone & 239 & 66.9 \\
\hline Dexmedetomidine + midazolam & 118 & 33.1 \\
\hline \multicolumn{3}{|l|}{ Adjuvants } \\
\hline Propofol & 56 & 15.7 \\
\hline Fentanyl & 353 & 98.9 \\
\hline \multicolumn{3}{|l|}{ Complications of sedation/mechanical ventilation } \\
\hline Healthcare-associated infection & 100 & 28.0 \\
\hline Pneumonia & 51 & 14.3 \\
\hline Delirium & 32 & 9.0 \\
\hline Tracheostomy & 26 & 7.3 \\
\hline Urinary tract infection & 19 & 5.3 \\
\hline Bacteremia & 10 & 2.8 \\
\hline Other infections & 20 & 5.6 \\
\hline Hospitalization variables & Mean (SD) & Median (IQR) \\
\hline Days of hospitalization-total & $13.1(13.9)$ & $9(3-19)$ \\
\hline Days of hospitalization in ICU & $9.1(11.1)$ & $6(2-13)$ \\
\hline Days of mechanical ventilation-total & $7.5(10.5)$ & $4(1.5-11)$ \\
\hline Days from the start of mechanical ventilation to discharge & $11.2(14.0)$ & $7(2-15)$ \\
\hline Number of extubation attempts (live) & $1.5(1.3)$ & $1(1-1)$ \\
\hline Clinical parameters-mechanical ventilation & Pre-sedation mean (SD) & $\begin{array}{l}\text { Post-sedation } \\
\text { mean (SD) }\end{array}$ \\
\hline Heart rate, $\mathrm{bpm}^{\mathrm{a}}$ & $92.8(25.1)$ & $90.8(25.3)$ \\
\hline Respiratory rate, $\mathrm{bpm}^{\mathrm{b}}$ & $20.4(7.3)$ & $18.9(5.1)$ \\
\hline Systolic blood pressure, $\mathrm{mmHg}$ & $126.5(34.6)$ & $120.5(35.1)$ \\
\hline Diastolic blood pressure, $\mathrm{mmHg}$ & $68.8(21.1)$ & $66.0(19.3)$ \\
\hline Mean arterial pressure, $\mathrm{mmHg}$ & $88.0(23.4)$ & $84.1(22.7)$ \\
\hline Hemoglobin, g/dL & $12.1(2.7)$ & $11.5(2.6)$ \\
\hline Hematocrit, \% & $36.8(8.7)$ & $35.9(8.2)$ \\
\hline Creatinine, $\mathrm{mg} / \mathrm{dL}$ & $1.9(2.8)$ & $1.7(2.1)$ \\
\hline
\end{tabular}

$I C U$ intensive care unit, $I Q R$ interquartile range, $S D$ standard deviation

${ }^{\text {a } B e a t s ~ p e r ~ m i n u t e ~}$

${ }^{\mathrm{b}}$ Breaths per minute

A significant association was observed between the use of vasopressors and a decrease in the likelihood of survival. The literature on the relationship between vasopressors and mortality is conflicting, although there is evidence of a deleterious effect of the need for vasopressors [27]. A meta-analysis published by Belletti et al., which included 177 studies evaluating the impact of using vasopressors/inotropes in critically ill patients, did not find an increase in mortality ( $p$ for effect $=0.23$ ) but actually reported decreases in mortality in treated patients of certain subgroups, particularly those with sepsis ( $p$ for effect $=0.02$ ), and in the group of studies that reported mortality at 1 month $(p$ for effect $=0.04$ ) [28], contrasting with what was found in this study. However, it is important to consider that the severity, as well as the progression of the disease, leads to the implementation of additional therapeutic strategies that seek the well-being and recovery of the patient. However, in some cases despite these efforts it will inevitably lead to death; therefore, the 
Table 4 Variables associated with the probability of being alive at discharge in patients undergoing invasive mechanical ventilation admitted to an intensive care unit in Colombia, 2017-2018

\begin{tabular}{|c|c|c|c|c|c|}
\hline Variables $(N=357)$ & $n(\%)^{\mathrm{a}}$ & $\begin{array}{l}\text { Alive at discharge } \\
(n=122) \\
n(\%)\end{array}$ & Beta & $\begin{array}{l}\text { Adjusted HR } \\
(95 \% \mathrm{CI})\end{array}$ & $p$-Value \\
\hline \multicolumn{6}{|l|}{ Clinical features } \\
\hline Age in years (quantitative) & NA & NA & -0.22 & $0.97(0.96-0.99)$ & 0.010 \\
\hline Admission with septic shock & $137(38.3)$ & $30(24.6)$ & -1.21 & $0.29(0.18-0.48)$ & $<0.001$ \\
\hline $\begin{array}{l}\text { Diabetes mellitus (comorbid- } \\
\text { ity) }\end{array}$ & $100(28.0)$ & $25(20.5)$ & -0.55 & $0.57(0.33-0.98)$ & 0.040 \\
\hline \multicolumn{6}{|l|}{ Complications } \\
\hline Required tracheostomy & $26(7.3)$ & $14(11.5)$ & -0.73 & $0.48(0.24-0.92)$ & 0.029 \\
\hline Healthcare-associated infection & $100(28.0)$ & $47(38.5)$ & -0.661 & $0.51(0.33-0.80)$ & 0.003 \\
\hline \multicolumn{6}{|l|}{ Medicines } \\
\hline Systemic corticosteroids & $243(68.1)$ & $98(80.3)$ & 0.66 & $1.93(1.15-3.25)$ & 0.013 \\
\hline Vasopressors/inotropics & $315(88.2)$ & $95(77.9)$ & -1.01 & $0.36(0.22-0.59)$ & $<0.001$ \\
\hline
\end{tabular}

Hazard ratio (HR) adjusted for age, sex, admission for septic shock and other shocks, trauma, firearm and stab injury, hypertension, diabetes, hypothyroidism, delirium, tracheostomy, infection, use of sedation with dexmedetomidine + midazolam, and comedications with antiepileptics, antipsychotics, benzodiazepines, systemic corticosteroids, muscle relaxants and vasopressors/inotropics

${ }^{\text {a }}$ Proportion over the total sample associations involving drugs and survival or death can have confounding biases.

On the other hand, patients who received systemic corticosteroids had a greater chance of survival at discharge. Varying results have been reported by different studies, especially in mechanically ventilated patients $[29,30]$. The ADRENAL study, which evaluated the use of hydrocortisone in an invasively mechanically ventilated population with sepsis, did not find a significant difference in mortality between patients who used hydrocortisone and those who did not [29]. On the other hand, the APPROCCHSS study on the use of hydrocortisone combined with fludrocortisone in patients with sepsis in the ICU, in which $91.8 \%$ were on mechanical ventilation, found a significantly lower 90-day mortality (RR 0.88 ; 95\% CI $0.78-0.99$ ) as well as more vasopressor-free days with their use [30]. A meta-analysis published by Fang et al., which included 37 studies that analyzed the impact on mortality in patients with sepsis after the use of systemic corticosteroids, found a lower 28-day mortality in patients with pharmacological therapy (RR 0.9; 95\% CI 0.82-0.98) and a lower ICU mortality (RR 0.85; 95\% CI 0.77-0.94), although no association was found with 90-day mortality (RR 0.94; 95\% CI 0.85-1.03) [31].

For patients with trauma, a benefit has also been found from the use of systemic corticosteroids, mainly through the reduced risk of presenting nosocomial pneumonia, but they may trigger other complications, such as sepsis, which thus increases the risk of mortality $[5,16]$. This beneficial effect is due to the improvement of corticosteroid deficiency associated with critical illness, which is present in up to $65 \%$ of these patients [32]. In the HYPOLYTE study, which evaluated the effects of hydrocortisone therapy in trauma patients, this drug significantly reduced the incidence of nosocomial pneumonia, ICU stay, and the duration of treatment with vasopressors [33].

Finally, no significant differences were found in the survival of patients who received dexmedetomidine compared with those who did not. Different data have been reported in this regard in multiple clinical contexts [34, 35]: A Cochrane meta-analysis evaluating the impact of dexmedetomidine versus traditional sedatives on mortality in critically ill patients found no significant association between its use and mortality [34]. On the other hand, a meta-analysis published by Zhang et al., which included eight studies, evaluated the outcomes of patients who received dexmedetomidine in the context of sepsis and found a decrease in ICU mortality in those who received it with respect to those who did not (RR 0.44; 95\% CI 0.23-0.84) [35].

Certain limitations should be considered when interpreting our results. Our data were obtained from a group of patients from a single ICU, so the findings may not be extrapolated to other places. In addition, the records were obtained from clinical histories, and it is not possible to know the full context that led to the prescription of certain medications, such as vasopressors, antibiotics, and corticosteroids. Mortality at 30 or 90 days was not evaluated, so a comprehensive comparison with international studies was not possible. 


\section{Conclusion}

We can conclude that advanced age, a diagnosis of septic shock or diabetes mellitus at admission, complications such as infections, and receiving vasopressors/inotropes conferred a higher risk of dying in the ICU, while the use of systemic corticosteroids was associated with an increased probability of survival in different clinical contexts. These results can help ICU physicians expand their knowledge of the risks that can lead to fatal outcomes in this group of patients.

Acknowledgements To Soffy Claritza López for her work in obtaining the database and to Dr Carlos Sanchez for managing the acquisition of medical records and Fundación Hospital San Jose de Buga for their support in the development of this research.

\section{Declarations}

Funding This study did not receive funding.

Conflicts of interest/Competing interests The authors declare no conflicts of interest.

Ethics approval The protocol was approved by the Bioethics Committee of the Universidad Tecnológica de Pereira in the category of riskfree research. The ethical principles established by the Declaration of Helsinki were respected. Reference number: 01-220719.

Consent to participate Not applicable, this is a retrospective observational study.

Availability of data and material protocolos.io.

Code availability https://dx.doi.org/10.17504/protocols.io.bpkamkse.

Author contributions All authors contributed to the concept and research design of the study; LFVR contributed to the assembly of data and analysis; and all authors participated in the interpretation of results and contributed to manuscript development.

Open Access This article is licensed under a Creative Commons Attribution-NonCommercial 4.0 International License, which permits any non-commercial use, sharing, adaptation, distribution and reproduction in any medium or format, as long as you give appropriate credit to the original author(s) and the source, provide a link to the Creative Commons licence, and indicate if changes were made. The images or other third party material in this article are included in the article's Creative Commons licence, unless indicated otherwise in a credit line to the material. If material is not included in the article's Creative Commons licence and your intended use is not permitted by statutory regulation or exceeds the permitted use, you will need to obtain permission directly from the copyright holder. To view a copy of this licence, visit http://creativecommons.org/licenses/by-nc/4.0/.

\section{References}

1. Leyes L, Verga F, Godino M, Barbato M. Tiempo de deterioro clínico extra UCI y sobrevida: una casuística. Rev Méd Urug. 2016;32(4):281-8.

2. López-Herce J, Carrillo Á. Ventilación mecánica: indicaciones, modalidades y programación y controles. Anales de Pediatría Continuada. 2008;6(6):321-9. https://doi.org/10.1016/S16962818(08)75597-5.

3. Mehta AB, Syeda SN, Wiener RS, Walkey AJ. Epidemiological trends in invasive mechanical ventilation in the United States: a population-based study. J Crit Care. 2015;30(6):1217-21. https:// doi.org/10.1016/j.jcrc.2015.07.007.

4. Alomía Ft D, Coral Ft M, Ortegón Ft S, Soto MdR, Muñoz V. Factores de riesgo asociados con la extubación fallida en pacientes adultos de una unidad de cuidados intensivos de la ciudad de Cali. Rev Cienc Salud. 2017;15(2):237-46.

5. Garay Z, Vera A, Pitta N, Bianco H, Ayala C, Almada P, et al. Impacto de las Neumonías Asociadas a la Ventilación Mecánica en la Mortalidad en una Unidad de Cuidados Intensivos Adultos. Rev Inst Med Trop. 2018;13(1):24-31. https://doi.org/10.18004/ imt/201813124-31.

6. Society of Critical Care Medicine. Critical Care Statistics. Society of Critical Care Medicine-The Intensive Care Professionals. 2019. https://www.sccm.org/Communications/Critical-Care-Stati stics. Accessed Jul 2020.

7. Tan E, Song J, Deane AM, Plummer MP. Global impact of coronavirus disease 2019 infection requiring admission to the ICU: a systematic review and meta-analysis. Chest. 2020. https://doi.org/ 10.1016/j.chest.2020.10.014.

8. Armstrong RA, Kane AD, Kursumovic E, Oglesby FC, Cook TM. Mortality in patients admitted to intensive care with COVID-19: an updated systematic review and meta-analysis of observational studies. Anaesthesia. 2021. https://doi.org/10.1111/anae.15425.

9. Arango-Isaza D, Velásquez-Duran M, Franco-Mesa C, CalleCorrea E, Jaramillo-Hurtado M, Ángel-Mejía V, et al. What happens to quality of life after the intensive care unit? Cross-sectional observational study. Rev colomb anestesiol. 2019;47(1):32-40. https://doi.org/10.1097/CJ9.0000000000000085.

10. Fialkow L, Farenzena M, Wawrzeniak IC, Brauner JS, Vieira SRR, Vigo A, et al. Mechanical ventilation in patients in the intensive care unit of a general university hospital in southern Brazil: an epidemiological study. Clinics. 2016;71:144-51. https://doi. org/10.6061/clinics/2016(03)05.

11. Weigl W, Adamski J, Gorynski P, Kanski A, Hultström M. ICU mortality and variables associated with ICU survival in Poland: a nationwide database study. Eur J Anaesthesiol. 2018;35(12):94954. https://doi.org/10.1097/EJA.0000000000000889.

12. Sauro KM, Soo A, de Grood C, Yang MMH, Wierstra B, Benoit $\mathrm{L}$, et al. Adverse events after transition from ICU to hospital ward: a multicenter cohort study. Crit Care Med. 2020;48(7):946-53. https://doi.org/10.1097/CCM.0000000000004327.

13. Adhikari NK, Fowler RA, Bhagwanjee S, Rubenfeld GD. Critical care and the global burden of critical illness in adults. Lancet (London, England). 2010;376(9749):1339-46. https://doi.org/10. 1016/S0140-6736(10)60446-1.

14. Yao H, Jayaraman D, Shahin J. Association between initial ICU admission diagnosis and outcomes in chronic critically ill patients: a retrospective cohort study. Can J Respir Crit Care Sleep Med. 2019. https://doi.org/10.1080/24745332.2019.1682953.

15. Chávez-Vivas M, Del Cristo-Martínez A, Tascón AJ. Características epidemiológicas de pacientes con diagnóstico de sepsis y choque séptico en un hospital de la ciudad de Cali, Colombia. Acta Méd Costarric. 2018;60(4):150-6. 
16. Guerra JJ, Posada-Upegui JC, Giraldo-Salazar OL. Morbimortalidad en trauma ortopédico de alta energía: estudio descriptivo retrospectivo. Rev Fac Nac Salud Pública. 2018;36(2):28-36. https://doi.org/10.17533/udea.rfnsp.v36n2a05.

17. van Vught LA, Holman R, de Jonge E, de Keizer NF, van der Poll T. Diabetes is not associated with increased 90-day mortality risk in critically ill patients with sepsis. Crit Care Med. 2017;45(10):e1026-35. https://doi.org/10.1097/CCM.00000 00000002590 .

18. Siegelaar SE, Hickmann M, Hoekstra JBL, Holleman F, DeVries $\mathrm{JH}$. The effect of diabetes on mortality in critically ill patients: a systematic review and meta-analysis. Crit Care (London, England). 2011;15(5):R205-R. https://doi.org/10.1186/cc10440.

19. Guidet B, Leblanc G, Simon T, Woimant M, Quenot JP, Ganansia $\mathrm{O}$, et al. Effect of systematic intensive care unit triage on longterm mortality among critically ill elderly patients in France: a randomized clinical trial. JAMA. 2017;318(15):1450-9. https:// doi.org/10.1001/jama.2017.13889.

20. Atramont A, Lindecker-Cournil V, Rudant J, Tajahmady A, Drewniak N, Fouard A, et al. Association of age with short-term and long-term mortality among patients discharged from intensive care units in France. JAMA Netw Open. 2019;2(5):e193215. https://doi.org/10.1001/jamanetworkopen.2019.3215.

21. Fleischmann C, Scherag A, Adhikari NK, Hartog CS, Tsaganos T, Schlattmann $\mathrm{P}$, et al. Assessment of global incidence and mortality of hospital-treated sepsis. Current estimates and limitations. Am J Respir Crit Care Med. 2016;193(3):259-72. https://doi.org/10. 1164/rccm.201504-07810C.

22. Reinhart K, Daniels R, Kissoon N, Machado FR, Schachter RD, Finfer S. Recognizing sepsis as a global health priority-a WHO resolution. N Engl J Med. 2017;377(5):414-7. https://doi.org/10. 1056/NEJMp1707170.

23. De Jong A, Rolle A, Molinari N, Paugam-Burtz C, Constantin JM, Lefrant JY, et al. Cardiac arrest and mortality related to intubation procedure in critically ill adult patients: a multicenter cohort study. Crit Care Med. 2018;46(4):532-9. https://doi.org/10.1097/ CCM.0000000000002925.

24. Delbove A, Darreau C, Hamel JF, Asfar P, Lerolle N. Impact of endotracheal intubation on septic shock outcome: a post hoc analysis of the SEPSISPAM trial. J Crit Care. 2015;30(6):1174-8. https://doi.org/10.1016/j.jcrc.2015.08.018.
25. Rego Avila H, Delgado Rodríguez A, Vitón Castillo AA, Piñeiro Izquierdo S, Machado MO. Neumonía asociada a la ventilación mecánica en pacientes atendidos en una unidad de cuidados intensivos. Revista de Ciencias Médicas de Pinar del Río. 2020;24:29-36.

26. Vincent J-L, Rello J, Marshall J, Silva E, Anzueto A, Martin CD, et al. International study of the prevalence and outcomes of infection in intensive care units. JAMA. 2009;302(21):2323-9. https:// doi.org/10.1001/jama.2009.1754.

27. Dünser MW, Hasibeder WR. Sympathetic overstimulation during critical illness: adverse effects of adrenergic stress. J Intensive Care Med. 2009;24(5):293-316. https://doi.org/10.1177/08850 66609340519.

28. Belletti A, Castro ML, Silvetti S, Greco T, Biondi-Zoccai G, Pasin L, et al. The Effect of inotropes and vasopressors on mortality: a meta-analysis of randomized clinical trials. B J Anaesth. 2015;115(5):656-75. https://doi.org/10.1093/bja/aev284.

29. Venkatesh B, Finfer S, Cohen J, Rajbhandari D, Arabi Y, Bellomo $\mathrm{R}$, et al. Adjunctive glucocorticoid therapy in patients with septic shock. N Engl J Med. 2018;378(9):797-808.

30. Annane D, Renault A, Brun-Buisson C, Megarbane B, Quenot J-P, Siami S, et al. Hydrocortisone plus fludrocortisone for adults with septic shock. N Engl J Med. 2018;378(9):809-18.

31. Fang F, Zhang Y, Tang J, Lunsford LD, Li T, Tang R, et al. Association of corticosteroid treatment with outcomes in adult patients with sepsis: a systematic review and meta-analysis. JAMA Internal Med. 2019;179(2):213-23.

32. Asehnoune K, Vourc'h M, Roquilly A. Hormone therapy in trauma patients. Crit Care Clin. 2019;35(2):201-11.

33. Roquilly A, Mahe PJ, Seguin P, Guitton C, Floch H, Tellier AC, et al. Hydrocortisone therapy for patients with multiple trauma: the randomized controlled HYPOLYTE study. JAMA. 2011;305(12):1201-9.

34. Chen K, Lu Z, Xin YC, Cai Y, Chen Y, Pan SM. Alpha-2 agonists for long-term sedation during mechanical ventilation in critically ill patients. Cochrane Database Syst Rev. 2015;1(1):CD010269.

35. Zhang WQ, Xu P, Zhan XH, Zheng P, Yang W. Efficacy of dexmedetomidine for treatment of patients with sepsis: a meta-analysis of randomized controlled trials. Medicine. 2019;98(18):e15469. 